\title{
Branching Processes of High-Level Petri Nets
}

\author{
Victor Khomenko and Maciej Koutny \\ School of Computing Science, University of Newcastle \\ Newcastle upon Tyne NE1 7RU, U.K. \\ \{Victor.Khomenko, Maciej.Koutny\}@ncl.ac.uk
}

\begin{abstract}
In this paper, we define branching processes and unfoldings of high-level Petri nets and propose an algorithm which builds finite and complete prefixes of such unfoldings. The advantage of our method is that it avoids a potentially expensive translation of a high-level Petri net into a low-level one. The approach is conservative as all the verification tools employing the traditional unfoldings can be reused with prefixes derived directly from high-level nets. We show that this is often better than the usual explicit construction of the intermediate low-level net.
\end{abstract}

Keywords: Verification, model checking, high-level Petri nets, unfolding.

\section{Introduction}

A distinctive characteristic of reactive concurrent systems is that their sets of local states have descriptions which are both short and manageable, and the complexity of their behaviour comes from highly complicated interactions with the external environment rather than from complicated data structures and manipulations thereon. One way of coping with this complexity problem is to use formal methods and, especially, computer aided verification tools implementing model checking (see, e.g., 4 ) - a technique in which the verification of a system is carried out using a finite representation of its state space.

The main drawback of model checking is that it suffers from the state space explosion problem. That is, even a relatively small system specification can yield a very large state space. To cope with this, a number of techniques have been proposed, which can roughly be classified as aiming at a compact representation of the full state space of a reactive system, or at an explicit generation of its reduced (though sufficient for a given verification task) representation.

McMillan's (finite prefixes of) Petri Net unfoldings (see, e.g., [20|21]) rely on the partial order view of concurrent computation, and represent system's actions and local states implicitly, using an acyclic net. In view of the development of fast model checking algorithms employing unfoldings (1114]), the problem of efficiently building them is becoming increasingly important. Recently, 78, 10[12 15 22 addressed this issue - considerably improving the original McMillan's technique - but we feel that generating net unfoldings deserves further investigation. In particular, it is highly desirable to generalize this technique to 
more expressive formalisms, such as high-level (or 'coloured') Petri nets. This formalism allows one to model in quite a natural way many constructs of highlevel specification languages used to describe concurrent systems (see, e.g., [1, 9]). Though it is possible to translate a high-level net into a low-level one and then unfold the latter, it is often the case that the intermediate low-level net is much larger than the resulting prefix.

In this paper, we propose an approach which allows one to build the prefix directly from a high-level net. Such a method is often superior to the traditional one, involving the explicit construction of an intermediate low-level net.

Notation. A multiset over a set $X$ is a function $\mu: X \rightarrow \mathbb{N} \stackrel{\text { df }}{=}\{0,1,2, \ldots\}$ (any subset of $X$ may be viewed through its characteristic function as a multiset over $X)$. We denote $x \in \mu$ if $\mu(x) \geq 1$, and for two multisets over $X, \mu$ and $\mu^{\prime}$, we write $\mu \leq \mu^{\prime}$ if $\mu(x) \leq \mu^{\prime}(x)$ for all $x \in X$. $\varnothing$ denotes the empty multiset defined by $\varnothing(x) \stackrel{\text { df }}{=} 0$, for all $x \in X$. A finite multiset may be represented by explicitly listing its elements between the $\{\ldots\}$ brackets, e.g., $\{y, y, z\}$ denotes $\mu$ such that $\mu(y)=2, \mu(z)=1$ and $\mu(x)=0$, for $x \in X \backslash\{y, z\}$. The sum of two multisets $\mu$ and $\mu^{\prime}$ over $X$ is given by $\left(\mu+\mu^{\prime}\right)(x) \stackrel{\text { df }}{=} \mu(x)+\mu^{\prime}(x)$, the difference by $\left(\mu-\mu^{\prime}\right)(x) \stackrel{\text { df }}{=} \max \left\{0, \mu(x)-\mu^{\prime}(x)\right\}$, and the intersection by $\left(\mu \cap \mu^{\prime}\right)(x) \stackrel{\text { df }}{=}$ $\min \left\{\mu(x), \mu^{\prime}(x)\right\}$, for all $x \in X$. A multiset $\mu$ is finite if there are finitely many $x \in X$ such that $\mu(x) \geq 1$. In such a case, the cardinality of $\mu$ is defined as $|\mu| \stackrel{\text { df }}{=} \sum_{x \in X} \mu(x) .\{P(x) \mid x \in \mu\}$, where $\mu$ is a multiset and $P(x)$ is an object constructed from $x \in X$, will be used to denote the multiset $\mu^{\prime}$ such that $\mu^{\prime}(y) \stackrel{\text { df }}{=} \sum_{x \in X \wedge P(x)=y} \mu(x) \cdot y$, where $\mu(x) \cdot y$ is the multiset consisting of exactly $\mu(x)$ copies of $y$, e.g., $\left\{x^{2}+1 \mid x \in\{-1,0,0,1\}\right\}=\{1,1,2,2\}$. For a mapping $h: X \rightarrow Y$ and a multiset $\mu$ over $X$, we denote $h\{\mu\} \stackrel{\text { df }}{=}\{h(x)|x \in \mu|\}$.

\section{Low-Level Petri Nets}

In this section, we first present basic definitions concerning Petri nets, and then recall (see also 6 8 16]) notions related to net unfoldings.

A net (with weighted arcs) is a triple $N \stackrel{\text { df }}{=}(P, T, W)$ such that $P$ and $T$ are disjoint sets of respectively places and transitions, and $W$ is a multiset over $(P \times T) \cup(T \times P)$ called the weight function. A net $N$ is called ordinary if $W$ is a set; in such a case, $W$ can be considered as a flow relation on $(P \times T) \cup(T \times P)$. A marking of $N$ is a multiset $M$ over $P$, and the set of all markings of $N$ will be denoted by $\mathcal{M}(N)$. (Note that $M$ is finite whenever $P$ is.) We adopt the standard rules about drawing nets, viz. places are represented as circles, transitions as boxes, the weight function by arcs with the indicated weight (we do not draw arcs whose weight is 0 , and we do not indicate the weight if it is 1), and markings are shown by placing tokens within circles. The multisets $\boldsymbol{\bullet} z \stackrel{\mathrm{df}}{=}\{y \mid(y, z) \in W\}$ and $z^{\bullet} \stackrel{\text { df }}{=}\{y \mid(z, y) \in W\}$, denote the pre- and postset of $z \in P \cup T$. (Note that for an ordinary net, both ${ }^{\bullet} z$ and $z^{\bullet}$ are sets.) We will assume that $\bullet^{\bullet} \neq \varnothing \neq t^{\bullet}$, for every $t \in T$. 
A net system is a pair $\Sigma \stackrel{\text { df }}{=}\left(N, M_{0}\right)$ comprising a finite net $N=(P, T, W)$ and an initial marking $M_{0}$. A transition $t \in T$ is enabled at a marking $M$ if $\bullet t \leq M$. Such a transition can be fired, leading to the marking $M^{\prime} \stackrel{\text { df }}{=} M-\bullet t+t^{\bullet}$; we denote this by $M[t\rangle M^{\prime}$. The set of reachable markings of $\Sigma$ is the smallest (w.r.t. $\subset$ ) set $\mathcal{R} \mathcal{M}(\Sigma)$ containing $M_{0}$ and such that if $M \in \mathcal{R} \mathcal{M}(\Sigma)$ and $M[t\rangle M^{\prime}$, for some $t \in T$, then $M^{\prime} \in \mathcal{R} \mathcal{M}(\Sigma)$.

$\Sigma$ is $k$-bounded if, for every reachable marking $M$ and every place $p \in P$, $M(p) \leq k$, and safe if it is 1-bounded. Moreover, $\Sigma$ is bounded if it is $k$-bounded for some $k \in \mathbb{N}$. One can show that the set $\mathcal{R} \mathcal{M}(\Sigma)$ is finite iff $\Sigma$ is bounded.

Places $p_{1}, \ldots, p_{k}$ of a net system $\Sigma$ are mutually exclusive if no reachable marking puts tokens on more than one of them, i.e., for every $M \in \mathcal{R} \mathcal{M}(\Sigma)$, $M\left(p_{i}\right) \geq 1$ implies $M\left(p_{j}\right)=0$, for all $j \in\{1, \ldots, k\} \backslash\{i\}$.

Low-level branching processes. Two nodes (places or transitions), $y$ and $y^{\prime}$, of an ordinary net $N=(P, T, W)$ are in conflict, denoted by $y \# y^{\prime}$, if there are distinct transitions $t, t^{\prime} \in T$ such that $\bullet^{\bullet} \cap \bullet^{\prime} \neq \varnothing$ and $(t, y)$ and $\left(t^{\prime}, y^{\prime}\right)$ are in the reflexive transitive closure of the flow relation $W$, denoted by $\preceq$. A node $y$ is in self-conflict if $y \# y$.

An occurrence net is an ordinary net $O N \stackrel{\text { df }}{=}(B, E, G)$, where $B$ is the set of conditions (places), $E$ is the set of events (transitions) and $G$ is a flow relation, satisfying the following: $O N$ is acyclic (i.e., $\preceq$ is a partial order); for every $b \in B$, $|\bullet b| \leq 1$; for every $y \in B \cup E, \neg(y \# y)$ and there are finitely many $y^{\prime}$ such that $y^{\prime} \prec y$, where $\prec$ denotes the transitive closure of $G$. $\operatorname{Min}(O N)$ will denote the set of minimal (w.r.t. $\prec$ ) elements of $B \cup E$. The relation $\prec$ is the causality relation. Two nodes are concurrent, denoted $y$ co $y^{\prime}$, if neither $y \# y^{\prime}$ nor $y \preceq y^{\prime}$ nor $y^{\prime} \preceq y$.

A homomorphism from an occurrence net $O N=(B, E, G)$ to a net system $\Sigma$ is a mapping $h: B \cup E \rightarrow P \cup T$ such that: $h(B) \subseteq P$ and $h(E) \subseteq T$ (conditions are mapped to places, and events to transitions); for each $e \in E, h\{\bullet e\}=\bullet h(e)$ and $h\left\{e^{\bullet}\right\}=h(e)^{\bullet}$ (transition environments are preserved); $h\{\operatorname{Min}(O N)\}=M_{0}$ (minimal conditions are mapped to the initial marking); and for all $e, f \in E$, if $\bullet e=\bullet$ and $h(e)=h(f)$ then $e=f$ (there is no redundancy). A branching process of $\Sigma$ is a pair $\pi \stackrel{\text { df }}{=}(O N, h)$ such that $O N$ is an occurrence net and $h$ is a homomorphism from $O N$ to $\Sigma$.

If an event $e$ is such that $h(e)=t$ then we will often refer to it as being $t$-labelled. A branching process $\pi^{\prime}=\left(O N^{\prime}, h^{\prime}\right)$ of $\Sigma$ is a prefix of a branching process $\pi=(O N, h)$, denoted by $\pi^{\prime} \sqsubseteq \pi$, if $O N^{\prime}=\left(B^{\prime}, E^{\prime}, G^{\prime}\right)$ is a subnet of $O N=(B, E, G)$ containing all minimal elements and such that: if $e \in E^{\prime}$ and $(b, e) \in G$ or $(e, b) \in G$ then $b \in B^{\prime}$; if $b \in B^{\prime}$ and $(e, b) \in G$ then $e \in E^{\prime}$; and $h^{\prime}$ is the restriction of $h$ to $B^{\prime} \cup E^{\prime}$. For each $\Sigma$ there exists a unique maximal (w.r.t. $\sqsubseteq$ ) branching process $U n f_{\Sigma}^{\max }$, called the unfolding of $\Sigma$ (see [6]).

Sometimes it is convenient to start a branching process with a (virtual) initial event $\perp$, which has the postset $\operatorname{Min}(O N)$, empty preset, and no label; we will use such an event, without drawing it in figures or treating it explicitly in algorithms.

Configurations and cuts. A configuration of an occurrence net $O N$ is a set of events $C$ such that for all $e, f \in C, \neg(e \# f)$ and, for every $e \in C, f \prec e$ 
implies $f \in C$; since we assume the initial event $\perp$, we additionally require that $\perp \in C$. For every $e \in E$, the configuration $[e] \stackrel{\text { df }}{=}\{f \mid f \preceq e\}$ is called the local configuration of $e$, and $\langle e\rangle \stackrel{\mathrm{df}}{=}[e] \backslash\{e\}$ denotes the set of causal predecessors of $e$. Moreover, for a set of events $E^{\prime}$, we denote by $C \oplus E^{\prime}$ the fact that $C \cup E^{\prime}$ is a configuration and $C \cap E^{\prime}=\varnothing$. Such an $E^{\prime}$ is a suffix of $C$, and $C \oplus E^{\prime}$ is an extension of $C$.

The set of all finite (resp. local) configurations of a branching process $\pi$ is denoted by $\mathcal{C}_{\text {fin }}^{\pi}\left(\operatorname{resp} . \mathcal{C}_{l o c}^{\pi}\right)$, and we will drop the superscript $\pi$ if $\pi=U n f_{\Sigma}^{\max }$.

A set of events $E^{\prime}$ is downward-closed if all causal predecessors of the events in $E^{\prime}$ also belong to $E^{\prime}$. Such a set induces a unique branching process $\pi$ whose events are exactly the events in $E^{\prime}$, and whose conditions are the conditions adjacent to the events in $E^{\prime}$ (including $\perp$ ).

A set of conditions $B^{\prime}$ such that for all distinct $b, b^{\prime} \in B^{\prime}, b$ co $b^{\prime}$, is called a co-set. A cut is a maximal (w.r.t. $\subset$ ) co-set. Every marking reachable from $\operatorname{Min}(O N)$ is a cut.

Let $C$ be a finite configuration of a branching process $\pi$. Then the set $C u t(C) \stackrel{\text { df }}{=}\left(\bigcup_{c \in C} c^{\bullet}\right) \backslash\left(\bigcup_{c \in C}{ }^{\bullet} c\right)$ is a cut (note that $\left.\perp \in C\right)$; moreover, the multiset of places $\operatorname{Mark}(C) \stackrel{\text { df }}{=} h\{C u t(C)\}$ is a reachable marking of $\Sigma$, called the final marking of $C$. A marking $M$ of $\Sigma$ is represented in $\pi$ if there is $C \in \mathcal{C}_{\text {fin }}^{\pi}$ such that $M=\operatorname{Mark}(C)$. Every marking represented in $\pi$ is reachable in the original net system $\Sigma$, and every reachable marking of $\Sigma$ is represented in $U n f_{\Sigma}^{\max }$.

Complete prefixes of Petri net unfoldings. Though unfoldings are infinite whenever the original net systems have infinite runs, it turns out that often they can be truncated in such a way that the resulting prefixes, though finite, contain enough information to decide a certain behavioural property, e.g., deadlock freeness. We then say that the prefixes are complete for this property.

There exist several different methods of truncating Petri net unfoldings. The differences are related to the kind of information about the original unfolding one wants to preserve in the prefix, as well as to the choice between using only local configurations (which can improve the running time of an unfolding algorithm), or all finite configurations (which can result in a smaller prefix), to cut the unfolding. In [16], a uniform approach to truncating unfoldings, based on cutting contexts, was proposed.

Cutting contexts. For greater flexibility, the approach proposed in [16] is parametric. The first parameter determines the information to be preserved in a complete prefix (in the standard case, the set of reachable markings). The main idea there was to shift the emphasis from the reachable markings of $\Sigma$ to the finite configurations of $U n f_{\Sigma}^{\max }$. Formally, the information to be preserved in the prefix corresponds to the equivalence classes of some equivalence relation $\approx$ on $\mathcal{C}_{\text {fin }}$. The other two parameters are more technical: they specify under which circumstances an event can be designated as a cut-off event (intuitively, this means that all its causal successors in the full unfolding can be removed).

A cutting context is a triple $\Theta \stackrel{\text { df }}{=}\left(\approx, \triangleleft,\left\{\mathcal{C}_{e}\right\}_{e \in E}\right)$, where:

1. $\approx$ is an equivalence relation on $\mathcal{C}_{\text {fin }}$. 
2. $\triangleleft$, called an adequate order, is a strict well-founded partial order on $\mathcal{C}_{\text {fin }}$ refining $\subset$, i.e., $C^{\prime} \subset C^{\prime \prime}$ implies $C^{\prime} \triangleleft C^{\prime \prime}$.

3 . $\approx$ and $\triangleleft$ are preserved by finite extensions, i.e., for every pair of configurations $C^{\prime} \approx C^{\prime \prime}$, and for every suffix $E^{\prime}$ of $C^{\prime}$, there exists a finite suffix $E^{\prime \prime}$ of $C^{\prime \prime}$ such that: $C^{\prime \prime} \oplus E^{\prime \prime} \approx C^{\prime} \oplus E^{\prime}$, and if $C^{\prime \prime} \triangleleft C^{\prime}$ then $C^{\prime \prime} \oplus E^{\prime \prime} \triangleleft C^{\prime} \oplus E^{\prime}$. 4. $\left\{\mathcal{C}_{e}\right\}_{e \in E}$ is a family of subsets of $\mathcal{C}_{\text {fin }}$, i.e., $\mathcal{C}_{e} \subseteq \mathcal{C}_{\text {fin }}$, for all $e \in E$.

The main idea behind the adequate order is to specify which configurations will be preserved in the complete prefix; it turns out that all $\triangleleft$-minimal configurations in each equivalence class of $\approx$ will be preserved. The last parameter is needed to specify the set of configurations used later to decide whether an event can be designated as a cut-off event. For example, $\mathcal{C}_{e}$ may contain all finite configurations of $U n f_{\Sigma}^{\max }$, or, as it is usually the case in practice, only the local ones. We will say that a cutting context $\Theta$ is dense (saturated) if $\mathcal{C}_{e} \supseteq \mathcal{C}_{\text {loc }}$ (resp. $\left.\mathcal{C}_{e}=\mathcal{C}_{\text {fin }}\right)$, for all $e \in E$.

In practice, $\Theta$ is usually dense (or even saturated, see [10]), the adequate order is either McMillan's one (see [8]21]) or the total order proposed in [8], and at least the following equivalences $\approx$ have been shown to be of interest:

$-C^{\prime} \approx_{m a r} C^{\prime \prime}$ if $\operatorname{Mark}\left(C^{\prime}\right)=\operatorname{Mark}\left(C^{\prime \prime}\right)$. This is the most widely used equivalence (see 810,1220$]$ ). Note that the equivalence classes of $\approx_{\text {mar }}$ correspond to the reachable markings of $\Sigma$.

$-C^{\prime} \approx_{\text {code }} C^{\prime \prime}$ if $\operatorname{Mark}\left(C^{\prime}\right)=\operatorname{Mark}\left(C^{\prime \prime}\right)$ and $\operatorname{Code}\left(C^{\prime}\right)=\operatorname{Code}\left(C^{\prime \prime}\right)$, where Code is the signal coding function. Such an equivalence is used in [23] for unfolding Signal Transition Graphs (STGs) specifying asynchronous circuits.

$-C^{\prime} \approx_{\text {sym }} C^{\prime \prime}$ if $\operatorname{Mark}\left(C^{\prime}\right)$ and $\operatorname{Mark}\left(C^{\prime \prime}\right)$ are symmetric markings according to some suitable notion (see [513]). This equivalence is the basis of the approach aimed at reducing the size of prefix described in [5].

We will write $e \triangleleft f$ whenever $[e] \triangleleft[f]$. Clearly, $\triangleleft$ is a well-founded partial order on the set of events refining $\prec$. Hence, one can use the Noetherian induction for definitions and proofs, i.e., it suffices to define or prove something for an event under the assumption that it has already been defined or proven for all its $\triangleleft$-predecessors. In the rest of this section, we assume that the cutting context $\Theta$ is fixed.

A branching process $\pi$ of $\Sigma$ is complete w.r.t. a set $E_{\text {cut }}$ (see also [16]) of events of $U n f_{\Sigma}^{\max }$ if the following hold:

1. If $C \in \mathcal{C}_{\text {fin }}$, then there is $C^{\prime} \in \mathcal{C}_{\text {fin }}^{\pi}$ such that $C^{\prime} \cap E_{\text {cut }}=\varnothing$ and $C \approx C^{\prime}$.

2. If $C \in \mathcal{C}_{\text {fin }}^{\pi}$ is such that $C \cap E_{\text {cut }}=\varnothing$, and $e$ is an event such that $C \oplus\{e\} \in$ $\mathcal{C}_{\text {fin }}$, then $C \oplus\{e\} \in \mathcal{C}_{\text {fin }}^{\pi}$.

A branching process $\pi$ is complete if it is complete w.r.t. some set $E_{c u t}$.

Note that $\pi$ remains complete following the removal of all events $e$ for which $\langle e\rangle \cap E_{\text {cut }} \neq \varnothing$, after which the events from $E_{\text {cut }}$ (usually referred to as cut-off events) will be either maximal events of the prefix or not in the prefix at all. Note also that the last definition depends only on the equivalence $\approx$, and not on the other components of the cutting context. 
For the relation $\approx_{m a r}$, each reachable marking is represented by a configuration in $\mathcal{C}_{\text {fin }}$ and, hence, also by a configuration in $\mathcal{C}_{\text {fin }}^{\pi}$, provided that $\pi$ is complete. This is what is usually expected from a correct prefix. Moreover, the definition of completeness implies that all firings enabled by the configurations from $\mathcal{C}_{\text {fin }}^{\pi}$ containing no events from $E_{\text {cut }}$ are preserved (see [16] for the explanation why this property is desirable).

Static cut-off events. Here we recall (see also [16]) the definition of static cut-off events. They are defined w.r.t. the whole unfolding, so that they are independent on an algorithm (hence the term 'static'), together with feasible events, which are precisely those events whose causal predecessors are not cutoff events, and as such must be included in the prefix determined by the static cut-off events.

The sets of feasible events, denoted by fsble $_{\Theta}$, and static cut-off events, denoted by cut $_{\Theta}$, of $U n f_{\Sigma}^{\max }$ are defined thus:

1. An event $e$ is a feasible event if $\langle e\rangle \cap c u t_{\Theta}=\varnothing$.

2. An event $e$ is a static cut-off event if it is feasible, and there is a configuration $C \in \mathcal{C}_{e}$ such that $C \subseteq f_{s b l e} \backslash$ cut $_{\Theta}, C \approx[e]$, and $C \triangleleft[e]$. Any $C$ satisfying these conditions will be called a corresponding configuration of $e$.

It turns out that, due to the well-foundedness of $\triangleleft, f s b l e_{\Theta}$ and cut $_{\Theta}$ are well-defined sets (see [16]). Since $\langle\perp\rangle=\varnothing, \perp \in f_{s} b l e_{\Theta}$ by the above definition. Furthermore, $\perp \notin$ cut $_{\Theta}$, since $\perp$ cannot have a corresponding configuration. Indeed, $[\perp]=\{\perp\}$ is the smallest (w.r.t. $\subset$ ) configuration, and so $\triangleleft$-minimal by the definition of a cutting context.

Canonical prefix. Once the feasible events are defined, the following notion arises quite naturally. The canonical prefix of $U n f_{\Sigma}^{\max }$ is the branching process $U n f_{\Sigma}^{\Theta}$ induced by $f_{s b l e}$. Thus $U n f_{\Sigma}^{\Theta}$ is uniquely determined by the cutting context $\Theta$. In [16], it is proven that the canonical prefix is always complete, and the conditions which guarantee its finiteness are investigated. Further in this paper we will show that all these results can be imported to the theory of branching processes of high-level Petri nets.

Algorithms for generating canonical prefixes. It turns out that canonical prefixes can be constructed by straightforward generalizations of the existing unfolding algorithms (see, e.g., 812]). The slicing algorithm from [12], parameterized by a cutting context $\Theta$, is shown in Figure 1 (The algorithm proposed in [8] is its special case.) It is assumed that the function $\operatorname{PotExt}(\pi)$ finds the set of possible extensions of a branching process $\pi$, according to the following. For a branching process $\pi$ of $\Sigma$, a possible extension is a pair $(D, t)$, where $D$ is a co-set in $\pi$ and $t$ is a transition of $\Sigma$, such that $h\{D\}={ }^{\bullet} t$ and $\pi$ contains no $t$-labelled event with preset $D$. We will take the pair $(D, t)$ as a $t$-labelled event having $D$ as its preset.

Compared to the standard unfolding algorithm in [8, the slicing algorithm has the following modifications in its main loop. A set of events $S l$, called a slice, is chosen on each iteration and processed as a whole, without taking or adding any events from or to pe. A slice must satisfy the following conditions:

- $S l$ is a non-empty subset of the current set of possible extensions pe. 


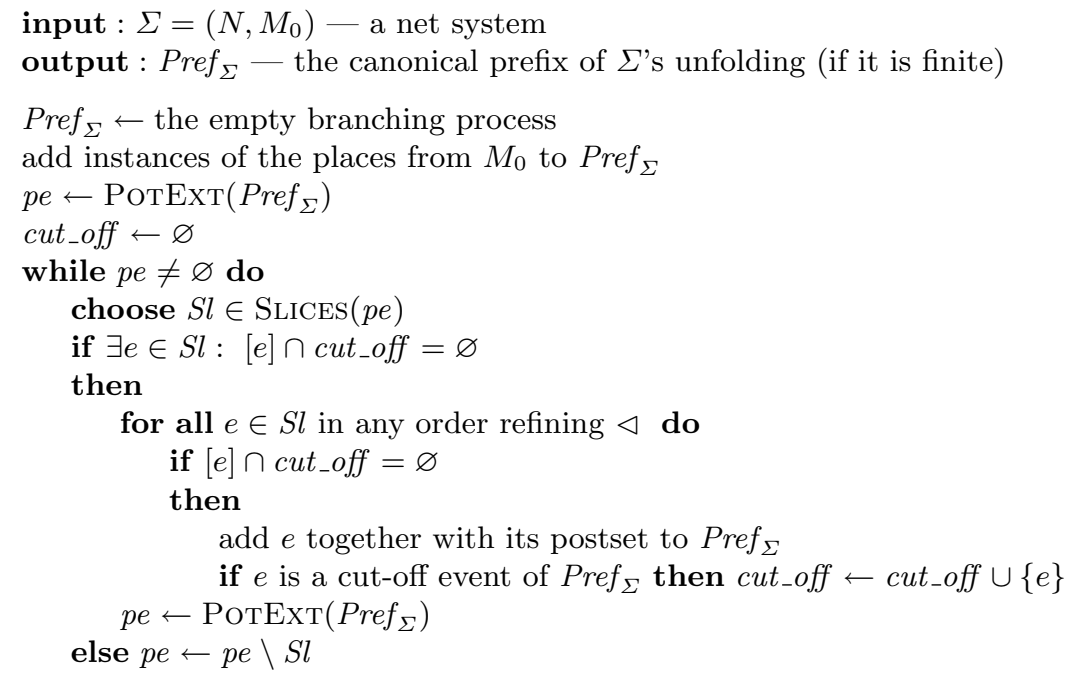

Fig. 1. Unfolding algorithm with slices ( $e$ is a cut-off event of $\operatorname{Pre} f_{\Sigma}$ if there is $C \in \mathcal{C}_{e}$

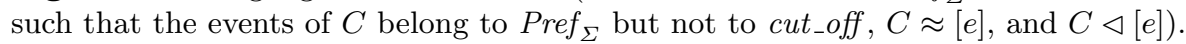

- For every $e \in S l$ and every event $f \triangleleft e$ of $U n f_{\Sigma}^{\max }, f \notin p e \backslash S l$ and $p e \cap\langle f\rangle=\varnothing$.

In particular, if $f \in p e$ and $f \triangleleft e$ for some $e \in S l$, then $f \in S l$. The set $\operatorname{SLICES}(p e)$ is chosen so that it is non-empty whenever pe is non-empty. Note that this algorithm, in general, exhibits more non-determinism than the one from [8]: it may be non-deterministic even if the order $\triangleleft$ is total. Since the events in the current slice can be processed independently, the slicing algorithm admits efficient parallelization (along the lines proposed in [12]). A crucial property of the slicing unfolding algorithm is that it generates the canonical prefix (see [12, 16]).

\section{$3 \quad$ High-Level Petri Nets}

In this paper we use M-nets (see [1]) as the main high-level Petri net model, as we believe that it is general enough to cover many other existing relevant formalisms. The full definition of M-nets can be found in 1]. Here, in order to match the presentation of low-level nets as closely as possible, we give a suitably adapted short description omitting those details which are not directly related to our purposes. In particular, 1 devotes a lot of attention to the composition rules, which are relevant only at the construction stage of an M-net, but not for model checking of an already constructed one.

M-nets. It is assumed that there exists a (finite or infinite) set Tok of elements (or 'colours') and a set $V A R$ of variable names, such that $T o k \cap V A R=\varnothing$. An $M$-net $N$ is a quadruple $N \stackrel{\text { df }}{=}(P, T, W, \iota)$ such that $P$ and $T$ are disjoint sets 
of respectively places and transitions, $W$ is a multiset over $(P \times V A R \times T) \cup$ $(T \times V A R \times P)$ of arcs, and $\iota$ is an inscription function with the domain $P \cup T$. It is assumed that, for every place $p \in P, \iota(p) \subseteq$ Tok is the type of $p$ and, for every transition $t \in T, \iota(t)$ is a boolean expression over $T o k \cup V A R$, called the guard of $t$. We assume that the types of all places are finite 1 In what follows, we assume that $N=(P, T, W, \iota)$ is a fixed M-net.

For a transition $t \in T$, let $\bullet \stackrel{\text { df }}{=}\left\{p^{v} \mid(p, v, t) \in W\right\}, t^{\bullet} \stackrel{\text { df }}{=}\left\{p^{v} \mid(t, v, p) \in W\right\}$, and $\operatorname{VAR}(t) \stackrel{\mathrm{df}}{=}\{v \mid(p, v, t) \in W\} \cup \operatorname{VAR}(\iota(t))$, where $\operatorname{VAR}(\iota(t))$ is the set of variables appearing in $\iota(t)$. A firing mode of $t$ is a mapping $\sigma: V A R(t) \rightarrow$ Tok such that $\sigma(v) \in \iota(p)$, for all $p^{v}$ in $\bullet t+t^{\bullet}$, and $\iota(t)$ evaluates to true under the substitution given by $\sigma$. (The notation $p^{v}$, similarly as $p^{x}$ and $t^{\sigma}$ used later on, is a shorthand for the pair $(p, v)$.)

We define the set of legal place instances as $\mathcal{P} \stackrel{\text { df }}{=}\left\{p^{x} \mid p \in P \wedge x \in \iota(p)\right\}$ and the set of legal firings as $\mathcal{T} \stackrel{\text { df }}{=}\left\{t^{\sigma} \mid t \in T\right.$ and $\sigma$ is a firing mode of $\left.t\right\}$. For every $t^{\sigma} \in \mathcal{T}$, we will also denote $t^{\sigma} \stackrel{\text { df }}{=}\left\{p^{\sigma(v)} \mid p^{v} \in{ }^{\bullet} t\right\}$ and $t^{\sigma \bullet} \stackrel{\text { df }}{=}\left\{p^{\sigma(v)} \mid p^{v} \in t^{\bullet}\right\}$. According to the definitions given below, all valid markings of an M-net will be composed of legal place instances, and its firing sequences will be composed of legal firings. Furthermore, the sets $\mathcal{P}$ and $\mathcal{T}$ will provide the basis for the construction of the low-level net corresponding to a high-level one.

A marking $M$ of $N$ is a multiset over $\mathcal{P}$. We will denote the set of all such markings by $\mathcal{M}(N)$. (Traditionally, a marking is a mapping which, to every place $p \in P$, associates a multiset over $\iota(p)$. Clearly, such a representation is equivalent to that we chose to use.)

The transition relation is a ternary relation on $\mathcal{M}(N) \times \mathcal{T} \times \mathcal{M}(N)$ such that a triple $\left(M, t^{\sigma}, M^{\prime}\right)$ belongs to it (denoted $\left.M\left[t^{\sigma}\right\rangle M^{\prime}\right)$ if $\bullet t^{\sigma} \leq M$ and $M^{\prime}=$ $M-\bullet t^{\sigma}+t^{\sigma} \bullet$. Note that $\sigma$ is a firing mode of $t$, which guarantees that $M^{\prime}$ is a valid marking of $N$.

M-net systems. An $M$-net system is a pair $\Upsilon \stackrel{\text { df }}{=}\left(N, M_{0}\right)$ comprising a finite M-net $N$ and an initial marking $M_{0}$. The set of reachable markings of an M-net system $\Upsilon$ is the smallest (w.r.t. $\subset$ ) set $\mathcal{R} \mathcal{M}(\Upsilon)$ containing $M_{0}$ and such that if $M \in \mathcal{R} \mathcal{M}(\Upsilon)$ and, for some $t^{\sigma} \in \mathcal{T}, M\left[t^{\sigma}\right\rangle M^{\prime}$ in $N$ then $M^{\prime} \in \mathcal{R} \mathcal{M}(\Upsilon)$.

An M-net system $\Upsilon$ is $k$-bounded if, for every marking $M \in \mathcal{R} \mathcal{M}(\Upsilon)$ and every $p^{x} \in \mathcal{P}, M\left(p^{x}\right) \leq k$; safe if it is 1-bounded; and bounded if it is $k$ bounded for some $k \in \mathbb{N}$. Moreover, $\Upsilon$ is strictly $k$-bounded if, for every marking $M \in \mathcal{R} \mathcal{M}(\Upsilon)$ and every place $p \in P,\left|\left\{|x| p^{x} \in M\right\}\right| \leq k$, and strictly safe if it is strictly 1-bounded. One can show that strictly $k$-bounded M-net systems are $k$ bounded, strictly safe ones are safe, and the set $\mathcal{R} \mathcal{M}(\Upsilon)$ is finite iff $\Upsilon$ is bounded. Note that according to the above definitions, a safe M-net system can have a reachable marking which places several tokens on the same place, provided that their 'colours' are all distinct. The rational behind our choice of the definition is that the low-level expansion (defined below) of an M-net system is safe iff the original M-net system is safe, and so the total adequate order proposed in 8 for safe net systems can be re-used (see the end of Section 4).

\footnotetext{
${ }^{1}$ In general, allowing infinite types yields a Turing-powerful model. Nevertheless, this restriction can be omitted in certain important cases (see Section 5).
} 

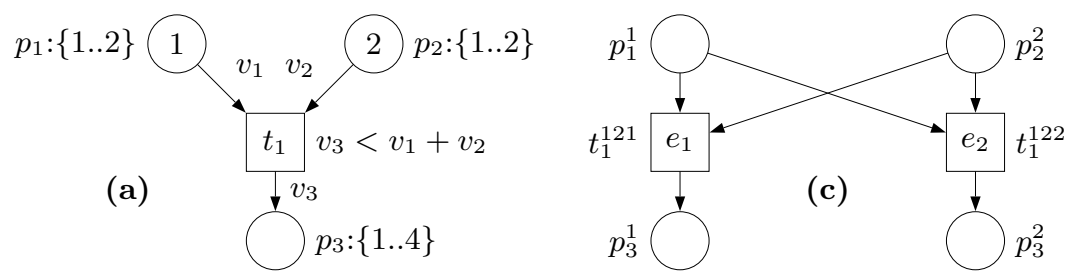

(b)

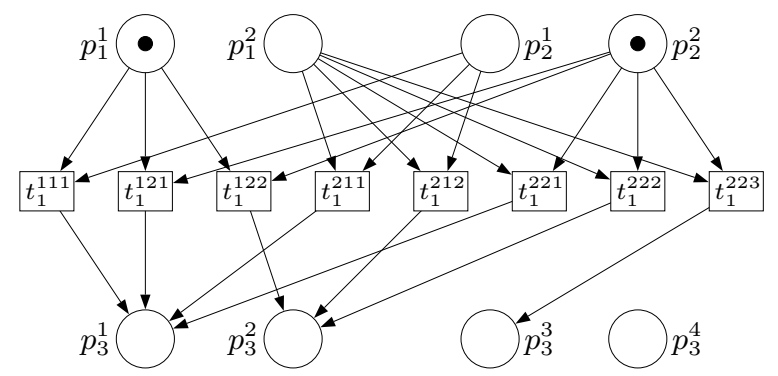

Fig. 2. An M-net system (a), its expansion (b), and its unfolding (c). Note that a firing mode $\sigma$ of $t$ is represented as a three-element string $\sigma\left(v_{1}\right) \sigma\left(v_{2}\right) \sigma\left(v_{3}\right)$.

Consider the M-net system shown in Figure 22(a). At the initial marking, $t_{1}$ can fire with the firing mode $\sigma \stackrel{\text { df }}{=}\left\{v_{1} \mapsto 1, v_{2} \mapsto 2, v_{3} \mapsto 1\right\}$ or $\sigma^{\prime} \stackrel{\text { df }}{=}\left\{v_{1} \mapsto 1, v_{2} \mapsto\right.$ $\left.2, v_{3} \mapsto 2\right\}$, consuming the tokens from $p_{1}$ and $p_{2}$ and producing respectively the token 1 or 2 on $p_{3}$. Formally, we have $\left\{p_{1}^{1}, p_{2}^{2}\right\}\left[t_{1}^{\sigma}\right\rangle\left\{p_{3}^{1}\right\}$ and $\left\{p_{1}^{1}, p_{2}^{2}\right\}\left[t_{1}^{\sigma^{\prime}}\right\rangle\left\{p_{3}^{2}\right\}$.

Transforming M-net systems into low-level nets. For each M-net it is possible to build an 'equivalent' low-level one. Such a transformation is called 'unfolding' in 1], but since this term is already used in this paper with a different meaning (see Section 2), we will use the term 'expansion' instead. The expansion $\mathcal{E}(N)$ of an M-net $N=(P, T, W, \iota)$ is a low-level net $\mathcal{E}(N) \stackrel{\text { df }}{=}\left(\mathcal{P}, \mathcal{T}, W^{\prime}\right)$ where $W^{\prime} \stackrel{\mathrm{df}}{=} \sum_{t^{\sigma} \in \mathcal{T}}\left(\left\{\left(p^{\sigma(v)}, t^{\sigma}\right) \mid(p, v, t) \in W\right\}+\left\{\left(t^{\sigma}, p^{\sigma(v)}\right) \mid(t, v, p) \in W\right\}\right)$. The expansion $\mathcal{E}(M)$ of a marking $M$ of $N$ is $M$ itself, i.e., $\mathcal{E}(M) \stackrel{\text { df }}{=} M$ (this is possible since there is no difference between the markings of $\mathcal{E}(N)$ and $N)$. Finally, the expansion of an M-net system $\Upsilon=\left(N, M_{0}\right)$ is defined as $\mathcal{E}(\Upsilon) \stackrel{\text { df }}{=}\left(\mathcal{E}(N), \mathcal{E}\left(M_{0}\right)\right)$ (see Figure 2(a,b)).

Proposition 1 ([1]). Let $N$ be an $M$-net, and $M^{\prime}, M^{\prime \prime} \in \mathcal{M}(N)$. Then $M^{\prime}\left[t^{\sigma}\right\rangle M^{\prime \prime}$ in $\Upsilon$ iff $M^{\prime}\left[t^{\sigma}\right\rangle M^{\prime \prime}$ in $\mathcal{E}(\Upsilon)$.

Proposition 2. Let $\Upsilon=\left(N, M_{0}\right)$ be an $M$-net system.

- For every $k \in \mathbb{N}, \mathcal{E}(\Upsilon)$ is $k$-bounded (safe) iff $\Upsilon$ is $k$-bounded (safe).

- If $\Upsilon$ is strictly safe and $p$ is a place of $\Upsilon$ then the places $p^{x}, x \in \iota(p)$, are mutually exclusive in $\mathcal{E}(\Upsilon)$.

Though, by Proposition 1, the expansion of an M-net system faithfully models the original system, the disadvantage of this transformation is that it usually 
yields a very large net. Moreover, the resulting net system is usually unnecessarily large, in the sense that it contains many places which cannot be marked and many dead transitions. This is so because the place types are usually overapproximations, and the transitions of the original M-net system may have many firing modes, only few of which are realized when executing the net from the initial marking. E.g., only two out of eight transitions of the expansion of the M-net system in Figure 2(a), shown in Figure 2(b), can actually fire. Therefore, though the M-net expansion is a neat theoretical construction, it is often impractical.

\section{Branching Processes of High-Level Nets}

In this section, we develop the main results of this paper, namely the notions of a branching process of an M-net system, the associated unfolding, and its canonical prefix. We also show that there is a strong correspondence between the branching processes of an M-net system and those of its expansion. This allows for importing many results from the theory of branching processes of low-level Petri nets.

A homomorphism from an occurrence net $O N=(B, E, G)$ to an M-net system $\Upsilon$ is a mapping $h: B \cup E \rightarrow \mathcal{P} \cup \mathcal{T}$ such that: $h(B) \subseteq \mathcal{P}$ and $h(E) \subseteq \mathcal{T}$ (conditions are mapped to legal place instances, and events to legal firings); for every $e \in E, h\{\bullet e\}=\bullet h(e)$ and $h\left\{e^{\bullet}\right\}=h(e)^{\bullet}$ (the environments of legal firings are preserved); $h\{\operatorname{Min}(O N)\}=M_{0}$ (minimal conditions are mapped to the initial marking); and for all $e, f \in E$, if $\bullet e=\bullet f$ and $h(e)=h(f)$, then $e=f$ (there is no redundancy). A branching process of $\Upsilon$ is a pair $\pi \stackrel{\text { df }}{=}(O N, h)$ such that $O N$ is an occurrence net and $h$ is a homomorphism from $O N$ to $\Upsilon$. (See Figure 2.)

This definition closely follows the definition of a (low-level) branching process of $\mathcal{E}(\Upsilon)$, and constitutes the main contribution of this paper. Because of this similarity, most of the definitions for branching processes of low-level net systems can now be lifted to branching processes of M-net systems. In particular, this is the case for the notions of a configuration, cut, final marking, the relation $\sqsubseteq$, cutting context, and the completeness of a prefix. Also, most of the results proven for branching processes of low-level Petri nets can also be lifted to branching processes of M-net systems. In particular, for each M-net system $\Upsilon$ there exist a unique (up to isomorphism) maximal (w.r.t. Б) branching process $U n f_{\Upsilon}^{\max }$ of $\Upsilon$, called the unfolding of $\Upsilon$. Moreover, for any cutting context $\Theta$ there exists unique canonical prefix $U n f_{\Upsilon}^{\Theta}$ (coinciding with $U n f_{\mathcal{E}(\Upsilon)}^{\Theta}$ ) of $U n f_{\Upsilon}^{\max }$, and the theory of canonical prefixes (see [16]) can be transferred without any changes.

It is straightforward to give an upper bound on the size of $U n f_{\Upsilon}^{\Theta}$, since the results of [8]16] regarding the size of the canonical prefix are still applicable. In particular, if the cutting context $\Theta=\left(\approx, \triangleleft,\left\{\mathcal{C}_{e}\right\}_{e \in E}\right)$ is dense, $\triangleleft$ is total, and $C^{\prime} \approx C^{\prime \prime} \Leftrightarrow \operatorname{Mark}\left(C^{\prime}\right)=\operatorname{Mark}\left(C^{\prime \prime}\right)$, then the number of non-cut-off events in $U n f_{\Upsilon}^{\Theta}$ does not exceed $|\mathcal{R} \mathcal{M}(\Upsilon)|$. 


\section{M-net Unfolding Algorithm}

Due to the results developed in the previous section, it is now possible to suggest a suitable modification of the standard unfolding algorithms, e.g., that in Figure 1, which is capable of building canonical prefixes of M-net unfoldings. It turns out that the only thing which has to be changed is the notion of a possible extension (so all the modifications are inside the POTExT function and thus are not visible in the top-level description of the algorithm).

For a branching process $\pi$ of an M-net system $\Upsilon$, a possible extension is a pair $\left(D, t^{\sigma}\right)$, where $D$ is a co-set in $\pi$ and $t^{\sigma}$ is a legal firing, such that $h\{D\}={ }^{\bullet} t$ and $\pi$ contains no $t^{\sigma}$-labelled event with the preset $D$. Similarly as in the low-level case, we will take the pair $\left(D, t^{\sigma}\right)$ as a new event of the prefix, with the preset $D$. After it is inserted into the prefix, its postset $D^{\prime}$ consisting of new conditions such that $h\left\{D^{\prime}\right\}=t^{\sigma \bullet}$ is also inserted.

It is worth noting that most of the existing heuristics aiming at speeding up the prefix generation can be applied. In particular, the total adequate order for safe net systems proposed in 8] can be used to unfold safe M-net systems. It is still adequate, since $U n f_{\Upsilon}^{\max }$ coincides with $U n f_{\mathcal{E}(\Upsilon)}^{\max }$ and the expansion of a safe M-net system is safe. Moreover, the concurrency relation (see [7!22]) can also be employed, even for non-safe systems. As for the preset trees (see [15]), they can be used without any modifications to unfold strictly safe M-net systems (and we work now on generalizing them to wider net classes).

It turns out that direct unfolding a high-level net not only avoids the generation of its (potentially, very large) expansion, but often is also more efficient than unfolding its expansion. Indeed, the most time-consuming part of the algorithm is computing the possible extensions (see [15]). Since one high-level transition usually corresponds to several low-level ones, less transitions have to be tried each time possible extensions are computed, which may lead to considerable savings in the running time.

It is often the case that the information about the firing mode of an event needs not be explicitly stored. Indeed, this information almost always can be discarded, since one is usually not interested what was the precise firing mode of a transition, as long as the consumed and produced tokens are the same.

An important extension of our approach allows for M-nets with places having infinite types. For example, it is often convenient to assign to a place the type $\mathbb{N}$ rather than $\{1, \ldots, n\}$, since $n$ might be not known in advance. Even when the set of reachable markings of such an M-net system is finite, its expansion is infinite and so of little use for model checking, whereas with our direct approach we still can build the canonical prefix and complete the verification. The only thing which needs to be ensured is that at any stage of prefix construction only a finite number of legal firings needs to be considered. This will be the case if, for every transition $t$ and every finite multiset $Z$ over $\mathcal{P}$, the set of all firing modes $\sigma$ of $t$ such that ${ }^{\bullet} t^{\sigma} \leq Z$ is both finite and computable.

Having built a canonical prefix, one can easily construct the refined version of the low-level expansion of the original M-net system, with unreachable places 

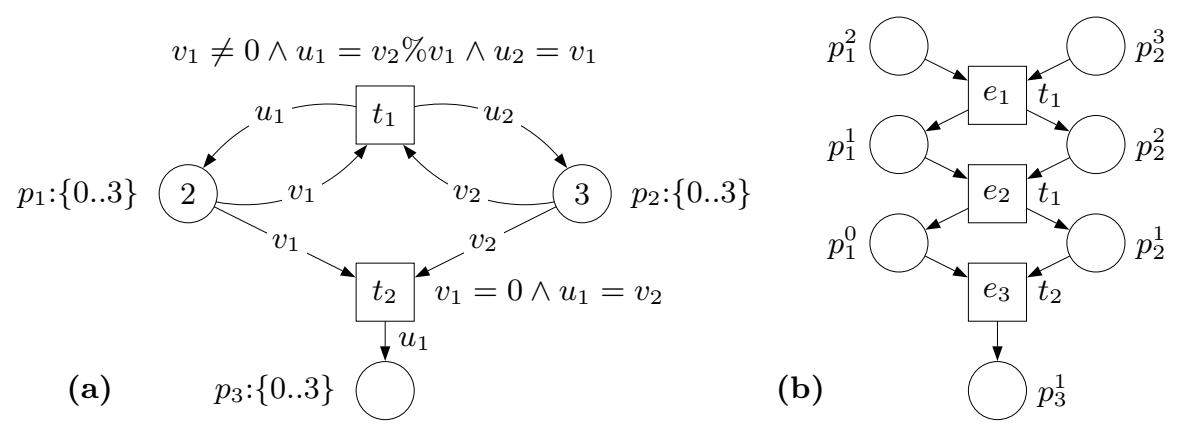

Fig. 3. An M-net system modelling Euclid's algorithm for computing the greatest common divisor of two non-negative integers (a), and its unfolding (b). Firing modes are not shown, but can easily be determined from events' presets and postsets.

and dead transitions removed. This may be important, e.g., for directly mapping a Petri net to a circuit simulating its behaviour (see, e.g., [3]).

Finally, it is worth mentioning that since our method constructs exactly the same prefix which would have been generated from the corresponding expansion of the M-net system, all the existing model checkers employing unfolding prefixes derived from low-level nets can be used without any changes when dealing with prefixes generated directly from M-net systems.

\section{Case Studies}

In this section, we compare our approach with the traditional one, viz. the unfolding of M-net expansions. We used the unfolding engine described in 1215 which after suitable modifications was able to unfold both low-level and highlevel nets. For building M-net expansions, we used the h12ll utility from the PEP tool (see [2]). The experiments were conducted on a PC with a Pentium ${ }^{T M}$ III/500MHz processor and 128M RAM.

The meaning of the columns in the tables is as follows (from left): the 'size' of the problem; the number of places and transitions in the original M-net system; the number of places and transitions in the corresponding expansion, together with the time required by the hl2ll utility to build the expansion; the number of conditions, feasible events, and cut-off events in the canonical prefix; the times (in seconds) required to unfold the expansion of the M-net system and the M-net system itself, respectively.

The first example is data-intensive, and so the traditional (via low-level nets) approach is extremely inefficient, whereas we expected our algorithm to perform well. The second example is control-intensive, so the M-net expansions are just slightly larger that the original M-nets. It was chosen to test the worst-case performance of our method relatively to unfolding of the low-level expansion. 
Greatest common divisor (GCD). An M-net simulating Euclid's algorithm for computing the greatest common divisor of two non-negative integers, together with its unfolding, is shown in Figure 3. In this net, $t_{1}$ fires until the number in $p_{1}$ becomes 0 , replacing the number in $p_{2}$ by that in $p_{1}$, and the number in $p_{1}$ by the residual of division of these two numbers. Then $t_{2}$ fires copying the result from $p_{2}$ to $p_{3}$. In our experiments, for each $N$ we computed the greatest common divisor of $F_{N}$ and $F_{N-1}$, where $F_{i}$ denotes the $i$-th Fibonacci's number (such numbers are known to produce the longest sequences of computational steps for Euclid's algorithm). The results of our experiments are summarized in Table1. From the structure of the M-net, it is easy to calculate that its expansion contains $3\left(F_{N}+1\right)$ places and $\left(F_{N}+1\right)^{2}$ transitions. These values are reported in the corresponding columns of the table, even though hl2ll failed to produce the expansions when they became large.

The experimental results show that for this example the high-level unfolding is clearly superior. Though the M-net expansion grows very quickly, the resulting prefix has only $2 N-1$ conditions and $N-1$ events. Therefore, our algorithm was able to build it for relatively large $N$ (we had to stop the experiments after $N=45$ since $F_{50}$ overflows 4 -bytes integer, but it is a limitation of the current implementation rather than of the method itself).

Mutual exclusion algorithm. The previous example was rather favourable for our algorithm, since the expansions of the M-net systems were very large. We therefore checked the performance of our approach in a totally opposite case, when the expansion of an M-net is relatively small. This happens when the transitions of the M-net are connected to few places and the cardinality of most place types is 1 . Such M-nets arise when modelling Lamport's mutual exclusion algorithm (see 1319]), which employs 'very small' atomic actions. We encoded it in the $B(P N)^{2}$ language supported by the PEP tool, and the corresponding experimental results are shown in Table 1 . As one can see, our algorithm performs almost as well as the algorithm for low-level nets. Though there is some overhead when computing transition guards and more complicated final states, it is relatively small, because the most time-consuming operation is computing the possible extensions of a current prefix. Moreover, this overhead becomes relatively smaller as the size of the prefix grows (it is just $0.5 \%$ for the last example in the table).

After the prefixes had been build, we verified using the efficient model checker described in 14 that the M-net system is deadlock free, and that the places corresponding to the critical sections of the processes are mutually exclusive. This was done without recompiling the model checker, since our unfolding algorithm generates prefixes which are indistinguishable from those generated by a lowlevel net unfolder from the corresponding expansions of the M-nets.

It is worth noting that in this example partial-order methods have advantage over the state-space ones. In [13, this mutual exclusion algorithm was verified for $N=3$ by building a reachability graph of the Petri net model and for $N=4$ by applying symmetry reductions. We managed to verify the case $N=4$ without applying symmetry reductions, using a PC with smaller memory (128M rather than $256 \mathrm{M}$ ), for a net which was generated from a relatively high-level description 
Table 1. Experimental results for the M-net systems simulating Euclid's GCD algorithm and Lamport's mutex algorithm.

\begin{tabular}{|r|rr|rrr|rrr|rr|}
\hline & \multicolumn{2}{|c|}{ M-net } & \multicolumn{4}{|c|}{ Expansion } & \multicolumn{3}{|c|}{ Unfolding } & \multicolumn{2}{|c|}{ Time[s] } \\
$\mathrm{N}$ & $|P|$ & $|T|$ & $|P|$ & $|T|$ & Time[s] & $|B|$ & $|E|$ & $\left|E_{\text {cut }}\right|$ & LL HL \\
\hline \hline 5 & 3 & 2 & 18 & 36 & $<1$ & 9 & 4 & 0 & $<1$ & $<1$ \\
10 & 3 & 2 & 168 & 3136 & 1 & 19 & 9 & 0 & 6 & $<1$ \\
15 & 3 & 2 & 1833 & $>10^{5}$ & - & 29 & 14 & 0 & - & $<1$ \\
20 & 3 & 2 & $>10^{4}$ & $>10^{7}$ & - & 39 & 19 & 0 & - & $<1$ \\
25 & 3 & 2 & $>10^{5}$ & $>10^{9}$ & - & 49 & 24 & 0 & - & $<1$ \\
30 & 3 & 2 & $>10^{6}>10^{11}$ & - & 59 & 29 & 0 & - & $<1$ \\
35 & 3 & 2 & $>10^{7}>10^{13}$ & - & 69 & 34 & 0 & $-<1$ \\
40 & 3 & 2 & $>10^{8}>10^{16}$ & - & 79 & 39 & 0 & $-<1$ \\
45 & 3 & 2 & $>10^{9}>10^{18}$ & - & 89 & 44 & 0 & $-<1$ \\
\hline
\end{tabular}

\begin{tabular}{|c|c|c|c|c|c|c|c|c|}
\hline \multirow[b]{2}{*}{$1 \mathrm{~V}$} & \multirow{2}{*}{$\begin{array}{l}\text { M-net } \\
|P||T|\end{array}$} & \multicolumn{2}{|c|}{ Expansion } & \multicolumn{3}{|c|}{ Unfolding } & \multicolumn{2}{|c|}{ Time[s] } \\
\hline & & $|P||T|$ & Time $[\mathrm{s}]$ & $|B|$ & $|E|$ & $\mid E_{c u}$ & 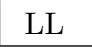 & $\mathrm{HL}$ \\
\hline 2 & $\begin{array}{ll}52 & 50\end{array}$ & 58 & & & & & & \\
\hline & $\begin{array}{ll}77 & 76\end{array}$ & 861 & & 4 & 12 & & 29 & 3 \\
\hline 4 & 0410 & 16236 & $<$ & 650 & 750 & 677 & $877^{\circ}$ & 391 \\
\hline
\end{tabular}

$\left(B(P N)^{2}\right.$ language) rather than built by hand. Moreover, our specification was not optimal since we had to replicate parts of the code, because $B(P N)^{2}$ does not currently have the goto operator (see [17 for more details). In principle, it is also possible to apply partial-order methods together with symmetry reductions (see [16]) to achieve even better results, but we have not implemented the combined method yet.

\section{Conclusions}

We defined branching processes and unfoldings of high-level Petri nets and proposed an algorithm which builds finite and complete prefixes. We established an important relation between the branching processes of a high-level net and those of its low-level expansion, viz. that the sets of their branching processes are the same, allowing us to import results proven for low-level nets. Among such results are the canonicity of the prefix for different cutting contexts, the usability of the total adequate order proposed in [8], and the parallel unfolding algorithm proposed in 12. Our approach is conservative in the sense that all the verification tools employing the traditional unfoldings can be reused with such prefixes. The conducted experiments demonstrated that it is, on one hand, superior to the traditional approach on data-intensive application, and, on the other hand, has the same performance on control-intensive ones. The full version of this paper $([17])$ contains a comparison with a similar work reported in [18].

Acknowledgements. This research was supported by an ORS Awards Scheme grant ORS/C20/4, and by EPSRC grants GR/M99293 and GR/M94366 (Movie). 


\section{References}

1. E. Best, H. Fleischhack, W. Fraczak, R. Hopkins, H. Klaudel, and E. Pelz: A Class of Composable High Level Petri Nets. ICATPN'1995, LNCS 935 (1995) 103-120.

2. E. Best and B. Grahlmann: PEP - more than a Petri Net Tool. TACAS'96, LNCS 1055 (1996) 397-401.

3. A. Bystrov and A. Yakovlev: Asynchronous Circuit Synthesis by Direct Mapping: Interfacing to Environment. ASYNC'02, IEEE Comp. Soc. Press (2002) 127-136.

4. E. M. Clarke, O. Grumberg, and D. Peled: Model Checking. MIT Press (1999).

5. J. -M. Couvreur, S. Grivet, and Denis Poitrenaud: Unfolding of Products of Symmetrical Petri Nets. ICATPN'2001, LNCS 2075 (2001) 121-143.

6. J. Engelfriet: Branching processes of Petri Nets. Acta Inf. 28 (1991) 575-591.

7. J. Esparza and S. Römer: An Unfolding Algorithm for Synchronous Products of Transition Systems. CONCUR'99, LNCS 1664 (1999) 2-20.

8. J. Esparza, S. Römer and W. Vogler: An Improvement of McMillan's Unfolding Algorithm. TACAS'96, LNCS 1055 (1996) 87-106. Full version: Formal Methods in System Design 20(3) (2002) 285-310.

9. H. Fleischhack, B. Grahlmann: A Petri Net Semantics for $B(P N)^{2}$ with Procedures. PDSE'97, IEEE Computer Society Press (1997) 15-27.

10. K. Heljanko: Minimizing Finite Complete Prefixes. CSEP'99, Workshop Concurrency, Specification and Programming (1999) 83-95.

11. K. Heljanko: Using Logic Programs with Stable Model Semantics to Solve Deadlock and Reachability Problems for 1-Safe Petri Nets. Fund. Inf. 37(3) (1999) 247-268.

12. K. Heljanko, V. Khomenko and M. Koutny: Parallelisation of the Petri Net Unfolding Algorithm. TACAS'02, LNCS 2280 (2002) 371-385.

13. K. Jensen: Colored Petri Nets. Basic Concepts, Analysis Methods and Practical Use. EATCS Monographs on Theoretical Computer Science (1992).

14. V. Khomenko and M. Koutny: LP Deadlock Checking Using Partial Order Dependencies. CONCUR'2000, LNCS 1877 (2000) 410-425.

15. V. Khomenko and M. Koutny: Towards An Efficient Algorithm for Unfolding Petri Nets. CONCUR'2001, LNCS 2154 (2001) 366-380.

16. V. Khomenko, M. Koutny, and V. Vogler: Canonical Prefixes of Petri Net Unfoldings. CAV'02, LNCS 2404 (2002) 582-595.

17. V. Khomenko and M.Koutny: Branching Processes of High-Level Petri Nets. Techn. Rep. CS-TR-763, Department of Computing Science, University of Newcastle (2002).

18. V. E. Kozura: Unfolding of Colored Petri Nets. Techn. Rep. 80, A. P. Ershov Institute of Informatics Systems (2000).

19. L. Lamport: A Fast Mutual Exclusion Algorithm. ACM Transactions on Computer Systems 5(1) (1987) 1-11.

20. K. L. McMillan: Using Unfoldings to Avoid State Explosion Problem in the Verification of Asynchronous Circuits. CAV'92, LNCS 663 (1992) 164-174.

21. K. L. McMillan: Symbolic Model Checking. PhD thesis, CMU-CS-92-131 (1992).

22. S. Römer: Entwicklung und Implementierung von Verifikationstechniken auf der Basis von Netzentfaltungen. PhD thesis, Technische Universitat Munchen (2000).

23. A. Semenov: Verification and Synthesis of Asynchronous Control Circuits Using Petri Net Unfolding. PhD Thesis, University of Newcastle upon Tyne (1997). 